Published in 2011, Biosensors \& Bioelectronics 26:2728-2732.

\title{
On chip real time monitoring of B-cells hybridoma secretion of immunoglobulin
} Sarah Milgram ${ }^{a}$, Sandra Cortes ${ }^{b}$, Marie-Bernadette Villiers ${ }^{b}$, Patrice Marche $^{b}$, Arnaud Buhot ${ }^{c}$, Thierry Livache ${ }^{a}$ \& Yoann Roupioz ${ }^{a *}$ Affiliation

${ }^{a}$ CREAB group, SPRAM laboratory UMR 5819 (CEA-CNRS-UJF), Institute for Nanosciences and Cryogeny (INAC), CEA-Grenoble, 38054 Grenoble, FRANCE

${ }^{\mathrm{b}}$ INSERM-UJF U823, Albert Bonniot Institute, Rond-point de la Chantourne, 38706 La Tronche cedex, FRANCE

${ }^{\mathrm{C}}$ Theory group, SPRAM laboratory UMR 5819 (CEA-CNRS-UJF), Institute for Nanosciences and Cryogeny (INAC), CEA-Grenoble, 38054 Grenoble, FRANCE

\section{Corresponding author}

Yoann.roupioz@cea.fr; Phone: +33 4387898 79; Fax: +33 438785145

\section{$\underline{\text { Abstract }}$}

The secretions of molecules by cells are of tremendous interest for both fundamental insights studies and medical purposes. In this study, we propose a new biochip-based approach for the instantaneous monitoring of protein secretions, using antibody production by $B$ lymphocytes cultured in vitro. This was possible thanks to the Surface Plasmon Resonance imaging (SPRi) of a protein biochip where antigen proteins (Hen Egg Lysozyme, HEL) were micro-arrayed along with series of control proteins. B cell hybridomas were cultured on the chip and the secretion of immunoglobulins (antibody) specific to HEL was monitored in realtime and detected within only few minutes rather than after a 30-60 minutes incubation with standard ELISA experiments. This fast and sensitive detection was possible thanks to the sedimentation of the cells on the biochip sensitive surface, where local antibody concentrations are much higher before dilution in the bulk medium. An other interesting feature of this approach for the secretion monitoring was the independence of the SPR response -after normalization- regarding to the density of the surface-immobilized probes. Such biosensor might thus pave the way to new tools capable of both qualitative and semiquantitative analysis of proteins secreted by other immune cells.

Keywords: Protein micro-array; Surface plasmon resonance imaging; Real-time detection; Cellular secretion; Blood cell 


\section{Introduction}

Inter-cellular communication is of primary importance in any living multi-cellular organism. In the specific case of blood cells, the inter-cell signaling cascades are mainly mediated by the extracellular secretion of proteins, which shall act as firing events for downstream cells (Hernandez-Fuentes et al. 2003), even though physical contact between cells may account in a much lesser extent. The monitoring of the secreting activity can be carried out either at the molecular level -by the labeling of soluble proteins followed by a colorimetric read-out (Enzyme-Linked Immunosorbent Assay, ELISA)- or at the cellular level by culturing cells on surfacesensitive plates where cells are washed off before labeling on surface-captured secretions (ELISPOT) (Czerkinsky et al. 1983). Other techniques have been engineered to label individual cells -in solutionvia their secreted products (Manz et al. 1995) thus enabling a flow cytometric analysis of cell hybridomas. Altogether, these approaches are based on end-point analysis, due to the labeling-washing steps prior to the readout, thus preventing any access to the kinetic insights of the secretion processes.

The recent developments of microtechnologies paved the way to cell-based analysis, where some biological activities can be monitored at the cellular level (El-Ali et al. 2006; Toner and Irimia 2005). For instance, the secreting activities of blood cells are specially studied to give better insights of the immune responses (Hernandez-Fuentes et al. 2003). To that end, biochips and biosensors dedicated to functional cell analysis are promising tools due to their reduced size, the access to parallelized assays and the small amounts of required biological starting material (Chen and Davis 2006). With the aim of monitoring such events at the cellular level, the capture and characterization of fluorescently labeled lymphocytes have been developed on a miniaturized cytometry platform (Zhu et al. 2008a) whereas other studies were done on microarrays dedicated to the detection of cellular metabolites after labeling and read-out (Jones et al. 2008; Zhu et al. 2008b).

Surface Plasmon Resonance imaging (SPRi) is an optical technique suitable for label-free and real-time analysis of biomolecule interactions. SPR-based sensors have previously been used to detect interactions between many different types of molecules like DNA, peptide or protein (Grosjean et al. 2005; Guedon et al. 2000). More recently, this method was used to detect living cells (Hide et al. 2002; Suraniti et al. 2007). In this way, Localized Surface Plasmon Resonance (LSPR) and GratingCoupled Surface Plasmon Resonance imaging (GCSPRi) allowed the label-free detection of specific cells or cellular metabolites (Endo et al. 2008; Jin et al. 2009). Although getting an increasing interest for a large variety of applications on biosensors, there are only few examples of SPR-based real-time monitoring of extra-cellular secretions. Peterson's group described the culture of adherent cells deposited on a layer of fibronectin, coated with serum proteins. Although rather sensitive, such experiment did not allow the identification of the proteins secreted in the extra-cellular medium and precipitating on the sensor surface (Peterson et al. 2009). Very recently, Stybayeva et al. presented a setup composed of two independent chambers, one dedicated to a subtype T cells capture and the other devoted to the cytokine SPR detection by flowing the culture medium collected from the first chamber (Stybayeva et al.).

In the present work, we describe a protein microarray suitable for both on chip cells culture and simultaneous specific analysis of proteins secreted from hybridoma. As a proof of concept, murine B cell hybridoma secreting HEL-specific immunoglobulin $G$ (IgG) were cultured on a chip where HEL-antigen was arrayed at different concentrations (along with negative controls to assess any non-specific signal). The multiplexed format of such microarray makes possible the simultaneous monitoring of the products secreted from a single culture well whose bottom is the SPR sensing surface, interacting with several specific probes (antigens) (Scheme 1). The SPRi detection allowed significant improvement of analysis times, by comparison with standard techniques such as ELISA, due to both the label-free process and the real-time monitoring of biological interactions at the vicinity of the cells.

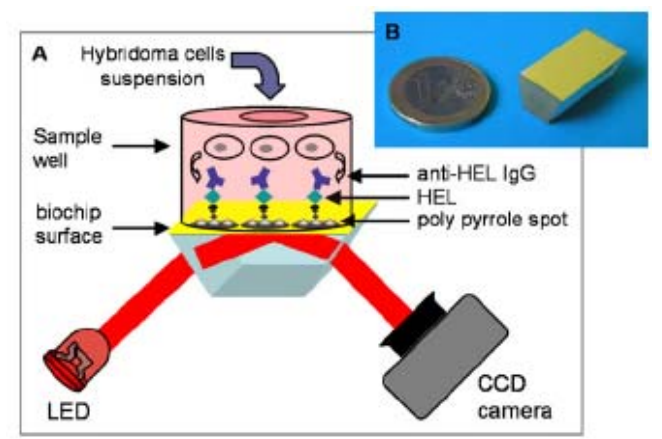

Scheme of the SPR imaging setup (A). Cells are loaded and cultured on a biochip where both antigen and control proteins have been electrochemically arrayed. Photograph of a gold-coated biochip (B).

\section{Materials and methods}

\subsection{Reagents and chemicals}

Cytochrome C, Bovine Serum Albumin (BSA), amino acids, Phosphate Buffer Saline (PBS), Penicillin and Streptomycin antibiotics were purchased from Sigma (Sigma-Aldrich, Saint Quentin-Falavier, France) as well as the culture media DMEM and RPMI, whereas the Fetal Bovine Serum (FSB) was from PAA Laboratoires (Les Mureaux, France). Pyrrole was purchased from Tokyo Casei (Japan), and was dissolved at a $1 \mathrm{M}$ concentration in dry acetonitrile (Sigma) in a glove box. 3,3',5,5'-tetramethylbenzidine (TMB) was purchased from Beckton Dickinson (BD Pharmingen, Pont de Claix, France), HEL lyophilized protein was from Merck (Paris, France). The StreptavidinPhycoerythrin used for the labeling of biotinylated molecules was from Invitrogen (Cergy Pontoise, France). The antibodies used as secondary agents - 
peroxidase-conjugated rabbit anti-mouse antibody, goat biotinylated anti-mouse antibody- were obtained from Sigma. All chemicals were used without further purification.

\subsection{Biochip preparation}

Gold covered prisms (50 $\mathrm{nm}$ thick gold layer over a $2 \mathrm{~nm}$ thick chromium layer) (Scheme 1B) were provided by GenOptics (Horiba ScientificGenOptics, Orsay, France). Biochips were electrochemically arrayed with proteins as described elsewhere (Grosjean et al. 2005). Briefly, Nhydroxysuccinimidyl 6-(pyrrolyl)-caproate (NHSpyrrole) was conjugated to proteins in $\mathrm{PBS}$ pH 7,4 overnight at $4^{\circ} \mathrm{C}$ using NHS-pyrrole/protein molar ratio of 10 . Conjugated proteins were then purified by ultracentrifugation on $3 \mathrm{kDa}$ MWCO membranes and diluted at different concentrations $(5,10$ and $20 \mu \mathrm{M})$ in a spotting buffer (50 mM phosphate buffer, $50 \mathrm{mM}$ $\mathrm{NaCl}$ and $10 \%$ glycerol, $\mathrm{pH} 6.8$ ) containing $20 \mathrm{mM}$ of free pyrrole. Proteins (HEL and Cytochrome $\mathrm{C}$ ) were then grafted on the chip by electro-copolymerization of free pyrrole and pyrrole-modified protein $(100 \mathrm{~ms}$, 2V ddp).

\subsection{Cells culture}

The mouse hybridoma cell line F10 producing monoclonal antibody anti-HEL (anti-Hen Egg Lysozyme) (Tello et al. 1993) was grown in DMEM supplemented with $10 \%$ Fetal Bovine Serum (FBS), non-essential amino acids ( $0.1 \mathrm{mM})$, sodium pyruvate $(1 \mathrm{mM}), 50 \mu \mathrm{M}$ 2-mercaptoethanol, $50 \mathrm{U} / \mathrm{mL}$ Penicillin and $50 \mu \mathrm{g} \cdot \mathrm{mL}^{-1}$ Streptomycin. The cultures were incubated at $37^{\circ} \mathrm{C}$ in a humidified $(95 \%)$ and $5 \% \mathrm{CO}_{2}$ incubator. Cells were centrifuged 5 minutes at $200 \mathrm{~g}$ prior to the experiments and cell pellets were re-suspended at $5.10^{5}$ cells. $\mathrm{mL}^{-1}$ in serum-free RPMI medium buffered with HEPES at $28 \mathrm{mM}$.

\subsection{ELISA titration of secreted anti-HEL IgG}

The secreting activities of cells used during SPRi experiments were characterized by ELISA assays. Aliquots $(1 \mathrm{~mL})$ of cellular suspension were incubated at $37^{\circ} \mathrm{C}$ in 24 -well plates. At different incubation times, the supernatant was collected and anti-HEL IgG secreted by the cells was quantified by ELISA according to standard protocols (Villiers et al. 1993). Briefly, the plates were coated with HEL $\left(10 \mu \mathrm{g} \cdot \mathrm{mL}^{-1}\right.$ in PBS$)$ for $2 \mathrm{~h}$ at $37^{\circ} \mathrm{C}$. Bound antibodies were revealed with peroxidase-conjugated rabbit anti-mouse IgG $(1 / 20,000)$ using TMB as substrate. Standard curves were plotted using calibrated solutions of monoclonal anti-HEL antibody purified from F10 cell line supernatants.

\subsection{SPRi detection of antibody secretion}

Cell incubations were carried out at $37^{\circ} \mathrm{C}$ in a PEEK culture well fitting the micro-arrayed face of the biochip (Scheme 1). After saturation of the chip surface with RPMI supplemented with $10 \%$ FBS, hybridoma B-cells, suspended in serum-free medium, were loaded into the well and their secreting activity was monitored by SPRi. Cells are not specifically bound on the biosensor surface but only loaded as a cells suspension. Then, cells quickly sediment on the surface (within few minutes) and thus get in the immediate vicinity of the grafted probes. Protein-protein interactions between secreted IgG and the sensor-grafted antigens induced variations of the refractive index near the gold surface, and thus triggering changes in reflected light intensities monitored using a 12-bit CCD (Guedon et al. 2000). The regions of interest were defined as spots areas where electropolymerization occurred. The mean feature size is about $300 \mu \mathrm{m}$ in diameter. The signal of every pixel included into this area was recorded, averaged with the other pixels of the same region of interest and plotted.

\subsection{Fluorescent detection of antigen-antibody} interactions

After the SPRi experiment was completed, the biochip was removed from the SPR imager and gently washed with PBS. F10 anti-HEL antibodies bound to the chip were labeled with a biotinylated anti-mouse antibody (10 minutes incubation at room temperature), followed by a StreptavidinPhycoerythrin conjugation step (10\% dilution in PBS, 5 minutes incubation). The fluorescent labeling of the spots was observed using an epi-fluorescence microscope (BX, Olympus) equipped with a Peltiercooled CCD camera (Hamamatsu, Japan).

\section{Results and discussion}

The mouse F10 hybridoma cell line was chosen for its ability to permanently secrete monoclonal anti-HEL antibodies. Biochips were thus functionalized with the HEL (antigen) along with cytochrome $\mathrm{C}$ which was chosen as a negative control due to a molecular weigh close to that of HEL protein. The microarraying of probe molecules was carried out by a localized electropolymerization of the proteins (spots c.a. $300 \mu \mathrm{m}$ in diameter) on a gold covered biochip (Grosjean et al. 2005). In such configuration, the gold film was used as a working electrode where both HEL-pyrrole conjugates (in solution at 5,10 and $20 \mu \mathrm{M}$ concentrations) and cytochrome C-pyrrole conjugates $(20 \mu \mathrm{M})$ could be grafted at different locations. Before the SPR imaging, cells were centrifuged and suspended in fresh culture medium to discard any anti-HEL antibodies secreted prior to the on-chip incubation. The cellular suspension was then loaded in a culture well designed to fit the biochip functionalized surface (Scheme 1). Binding between anti-HEL antibody secreted from cells contained in a single well- and HEL proteins grafted on the chip at different concentrations could be simultaneously detected by SPRi (Figure 1A). By choosing an antigen protein (HEL, about $15 \mathrm{kDa}$ ) as a probe recognized by an immunoglobulin $\mathrm{G}$ (about $150 \mathrm{kDa}$ ), we are in a favorable configuration for a sensitive target detection. The kinetics of cellular secretions released in the extra-cellular medium were recorded in realtime for eight hours by SPRi. Some discrete objects appeared randomly on HEL spots, corresponding to individual cells bound on the surface. This rare 
background response might be due to the presence of antibodies specific to the HEL antigen and bound to the cell membrane. Pixels intensities of these objects were monitored and were shown to not significantly affect the overall spot pixel averaging, with a contribution lower than five percent whatever the time of experiment (see supplemental data).

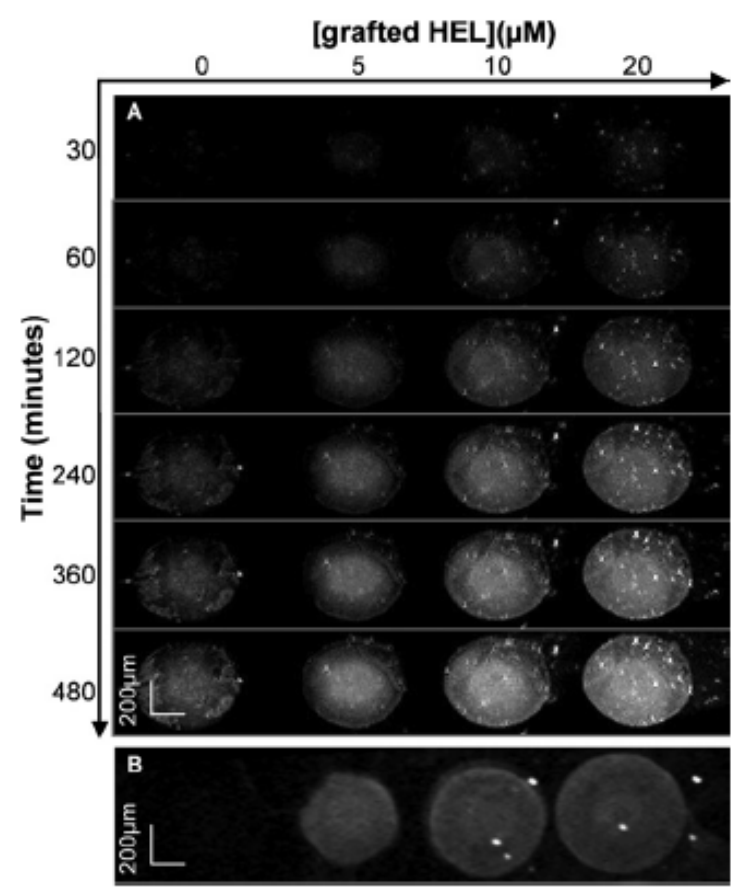

Figure 1: SPR image of a biochip arrayed with HEL $(0,5,10$ or $20 \mu \mathrm{M})$ after different incubation times with anti-HEL antibody secreting cells (A). The $0 \mu \mathrm{M}$ HEL spot corresponds to a cytochrome $C$ control spot $(20 \mu \mathrm{M}$, ) where no HEL was grafted.

Fluorescence image of the same spots observed by fluorescence microscopy after the SPR imaging experiment (B). Surface-bound anti-HEL antibodies were conjugated with anti-mouse IgG (Fc specific) and stained with Streptavidin-Phycoerythrin.

After incubation, surface bound proteins were fluorescently labeled by counter-staining of the mouse anti-HEL IgG with a goat anti-mouse antibody conjugated with biotin and followed by a StreptavidinPhycoerythrin recognition (Figure 1B). The much lower background signal observed on the end-point analysis is due to the molecular sandwich set-up where only target molecules bound to the surface are labeled by a secondary antibody specific to the HELimmunoglobulin.

Reflectivity variations of the HEL-grafted spots were plotted upon time by averaging the grey level of each pixel contained in a region of interest matching each individual spot. The plotted curves confirmed the highly specific recognition between antibodies and antigens detected only few minutes after cells loading in the culture well (Figure 2A). Once again, the specificity of the reaction was checked thanks to the cytochrome $\mathrm{C}$ control spots where no reflectivity drift could be observed thus assessing both the highly specific detection of antiHEL antibodies and a low background adsorption on control proteins. The background subtraction explains the differences of grey level images observed on the SPR images on figure $1 \mathrm{~A}$ versus the curves plotted in figure $2 \mathrm{~A}$ : even though the overall reflectivity level may drift upon time, subtraction of non-specific response (on polypyrrole spot) to the negative control cytochrome $\mathrm{C}$ spot exhibit only a weak variation during the time lap of the experiment.
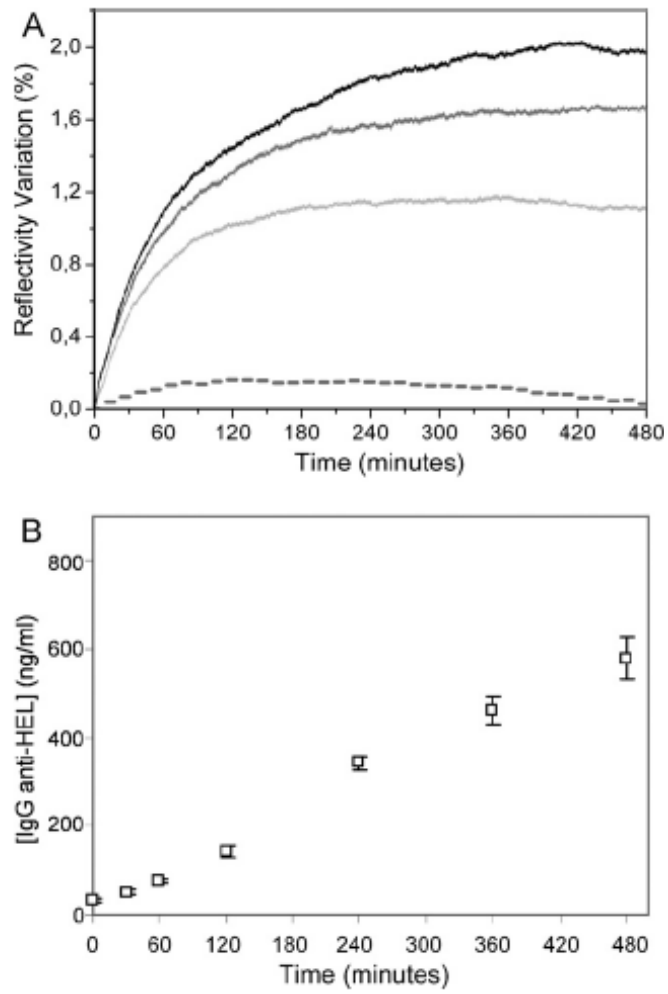

Figure 2: SPR monitoring of the reflectivity variations upon the incubation time. The biochip was arrayed with different spots polymerized with either cytochrome C $20 \mu \mathrm{M}$ (gray dotted line), HEL $5 \mu \mathrm{M}$ (light gray line), $10 \mu \mathrm{M}$ (dark gray line) or $20 \mu \mathrm{M}$ (black line) solutions (A). The concentrations refer to the concentration of the proteins in the solution prepared for the electrochemical polymerization of polypyrrole films on the biochip. Cells were loaded on chip at time 0 . The background signal corresponding to the unspecific response registered on polypyrrole spots was subtracted to the signal registered on the spots of interest. ELISA titration of anti-HEL antibodies contained in cellular supernatants after different incubation times (B).

Enzyme-linked Immunosorbent assay (ELISA) was used as a reference technique for the titration of antibody secretion. We exploited this endpoint colorimetric assay to compare the limits of detection (in terms of time required for the assessment of protein secretion) with SPR imaging performances. F10 hybridoma cells suspended in culture medium were loaded in multi-well plates and cultured at $37^{\circ} \mathrm{C}$ to ensure the anti-HEL secretion. On the contrary to SPR imaging where the detection occurs simultaneously with the cellular secretion (real-time monitoring), ELISA protocol requires the collection of sample aliquots for further labeling and titration (end-point analysis). Cellular supernatants were then collected at different incubation times and 
titrated according to standard ELISA protocols. Calibrated solutions of purified F10 anti-HEL antibody were used to get quantitative values of secreted antibodies. After a 30 minutes incubation, the concentration of $\operatorname{lgG}$ released in the supernatant was $51.3 \pm 5.6 \mathrm{ng} \cdot \mathrm{mL}^{-1}$ and increased linearly with time, reaching a concentration of $579.1 \pm 47.7 \mathrm{ng}^{-\mathrm{ml}^{-}}$ ${ }^{1}$ after eight hours of incubation (Figure 2B).

The SPRi curves, unlike the ELISA curve, showed a sharp signal increase for short incubation times (within one hour), with a significant response obtained faster than using ELISA test. In a second phase, the SPRi slopes decreased and reached a plateau. The three different observed plateaus were dependent on the concentration of monomer solutions used for probe grafting and reflect the saturation of grafted probes. Interestingly, by normalizing SPR kinetic curves against the different saturation plateau levels, we observed the perfect overlay of the curves whatever the concentration of probe antigens (see supplemental data). All the curves followed a linear dependency between time and reflectivity variation for short incubation times (less than one hour) and an exponential variation for longer incubation. A characteristic time value was extracted from the modeling of this data, and was shown to be consistent with independent ELISA experiments run in triplicate. More importantly, this characteristic time was shown to be independent on the density of grafted probes -in the range of grafted molecules shown here- but rather specific to the secretion phenomenon.

Although the typical detection limit of biomolecular interactions using SPR is in the nanomolar range (Scarano et al.), our approach showed significant improvements for the detection of secreted antibodies below nanomolar concentrations: focusing on the data acquired after an half-hour incubation, the ELISA titration evidenced an anti-HEL antibody concentration of about $50 \mathrm{ng} \cdot \mathrm{mL}^{-1}$, corresponding to $0.35 \mathrm{nM}$ in bulk medium, whereas SPR data showed a strong signal after only few minutes of incubation. Control SPR imaging experiment have been carried on with antiHEL antibodies at calibrated concentrations in the nanomolar range, and no significant response could be monitored (data not shown). Such results confirm the efficiency of this technique for the secretion detection in the close vicinity of the cell, rather than the detection of target molecules dissolved in the bulk medium (ELISA assays). This significant increase in sensitivity is mainly due to our experimental approach where living secreting cells are directly loaded onto the biochip and gently settled on the sensing surface. Because the SPR phenomenon is sensitive to any optical index change within few hundreds of nanometer above the gold layer, we were capable to explore the secretions in the intimate vicinity of the cells. Such focus on the direct environment of the cell gives access to locally high concentrations of secreted products which triggers a significant shift in the reflected light intensities. Besides this noticeable improvement of the limits of detection for cell secretion monitoring, the real-time detection drastically improves the time needed for the sample processing. Such shortening of the sample treatment time is possible thanks to the monitoring of the cells as they are secreting the molecules of interest, which is not the case with any label-based technique such as ELISA or ELISPOT assays (end-point analysis)

\section{Conclusion}

As a conclusion, we have successfully built a biochip suitable for the SPR based detection of cellular secretions. We have shown the interest of such cell-based technique to follow, in real-time, cellular secretions as the reflectivity variation detected within few minutes was shown to be specific to the secreting event, rather than to the grafted probe density. Besides a significant improvement of the processing time required by other conventional techniques, this approach saves several experimental steps (collection of cell suspensions, immuno-staining, washing and eventually colorimetric labeling and read-out), and does not require any labeling step involved in most other end-point techniques. Such improvements were possible by taking advantage of the evanescent-wave depth to explore the extra-cellular medium surrounding cells settled on the biochip surface. For the first time, we propose a one-step label-free method able to detect, in real time, secreting activities of living non-adherent cells cultured on a biochip. Moreover, the miniaturized sensor shows a great flexibility and is suitable for multi-target detection as several probes can be arrayed in a single biochip. The simultaneous analysis of different secreted products is currently in progress with primary cells capable of complex proteins secretion. This quick and easy method could provide a very useful tool for cellular phenotype analysis in different fields such as immunology where secreting activities are key biological events in the networking of blood non-adherent cells. 


\section{$\underline{\text { References }}$}

- Chen, D.S., Davis, M.M., 2006. Current opinion in chemical biology 10(1), 28-34.

- Czerkinsky, C.C., Nilsson, L.A., Nygren, H., Ouchterlony, O., Tarkowski, A., 1983. Journal of immunological methods 65(1-2), 109-121.

- $\quad$ El-Ali, J., Sorger, P.K., Jensen, K.F., 2006. Nature 442(7101), 403-411.

- Endo, T., Yamamura, S., Kerman, K., Tamiya, E., 2008. Analytica chimica acta 614(2), 182-189.

- Grosjean, L., Cherif, B., Mercey, E., Roget, A., Levy, Y., Marche, P.N., Villiers, M.B., Livache, T., 2005. Analytical biochemistry 347(2), 193-200.

- Guedon, P., Livache, T., Martin, F., Lesbre, F., Roget, A., Bidan, G., Levy, Y., 2000. Analytical chemistry 72(24), 6003-6009.

- Hernandez-Fuentes, M.P., Warrens, A.N., Lechler, R.I., 2003. Immunological reviews 196, 247-264.

- Hide, M., Tsutsui, T., Sato, H., Nishimura, T., Morimoto, K., Yamamoto, S., Yoshizato, K., 2002. Analytical biochemistry 302(1), 28-37.

- Jin, A., Ozawa, T., Tajiri, K., Obata, T., Kondo, S., Kinoshita, K., Kadowaki, S., Takahashi, K., Sugiyama, T., Kishi, H., Muraguchi, A., 2009. Nature Medicine 15(9), 1088-U1146.

- Jones, C.N., Lee, J.Y., Zhu, J., Stybayeva, G., Ramanculov, E., Zern, M.A., Revzin, A., 2008. Analytical chemistry 80(16), 6351-6357.

- Manz, R., Assenmacher, M., Pfluger, E., Miltenyi, S., Radbruch, A., 1995. Proceedings of the National Academy of Sciences of the United States of America 92(6), 1921-1925.

- Peterson, A.W., Halter, M., Tona, A., Bhadriraju, K., Plant, A.L., 2009. BMC cell biology 10, 16.

- Scarano, S., Mascini, M., Turner, A.P., Minunni, M., 2010. Biosensors \& bioelectronics 25(5), 957-966.

- Stybayeva, G., Kairova, M., Ramanculov, E., Simonian, A.L., Revzin, A., Colloids and surfaces 80(2), 251255.

- Suraniti, E., Sollier, E., Calemczuk, R., Livache, T., Marche, P.N., Villiers, M.B., Roupioz, Y., 2007. Lab Chip 7(9), 1206-1208.

- Tello, D., Goldbaum, F.A., Mariuzza, R.A., Ysern, X., Schwarz, F.P., Poljak, R.J., 1993. Biochemical Society transactions 21(4), 943-946.

- Toner, M., Irimia, D., 2005. Annual review of biomedical engineering 7, 77-103.

- Villiers, M.B., Gabert, F.M., Jacquier, M.R., Villiers, C.L., Colomb, M.G., 1993. Molecular immunology 30(2), 129-136.

- Zhu, H., Macal, M., Jones, C.N., George, M.D., Dandekar, S., Revzin, A., 2008a. Analytica chimica acta 608(2), 186-196.

- Zhu, H., Stybayeva, G., Macal, M., Ramanculov, E., George, M.D., Dandekar, S., Revzin, A., 2008b. Lab Chip 8(12), 2197-2205. 